\title{
Plantas medicinais conhecidas na zona urbana de Cajueiro da Praia, Piauí, Nordeste do Brasil
}

SANTOS, A.B.N.'; ARAÚJO, M. P. 1; SOUSA, R.S.2; LEMOS, J.R..*

Universidade Federal do Piauí - Av. São Sebastião 2819, Reis Velloso, Parnaíba-PI, 64.202-020. 2Universidade Federal Rural de Pernambuco(UFRPE), Laboratório de Etnobiologia Aplicada e Teórica (LEA). *Autor para correspondência: jelemos@ib.usp.br

RESUMO: A utilização de plantas com fins medicinais para tratamento, cura e prevenção de doenças é considerada uma das mais antigas formas de prática medicinal da humanidade. $O$ presente estudo teve como objetivo identificar as espécies de plantas medicinais usadas dentro da comunidade de Cajueiro da Praia, Piauí, bem como a parte da planta utilizada, o modo de preparo, a sua importância relativa, o valor de uso e o consenso em relação às propriedades terapêuticas das espécies citadas. Foram aplicadas 12 entrevistas semiestruturadas com especialistas locais indicados, utilizando o método de amostragem por "bola-de-neve" e a técnica de "turnê-guiada" para coleta das espécies citadas. Análises quantitativas tais como Valor de Uso, Importância Relativa e Fator de Consenso dos Informantes foram associadas à análises qualitativas. As coletas botânicas seguiram a metodologia usual. Foram citadas 43 espécies, distribuídas em 24 famílias botânicas, sendo Lamiaceae a mais representada em número de espécies (oito espécies), seguida de Euphorbiaceae, Fabaceae, Myrtaceae e Rutaceae (todas com três). Chenopodium ambrosioides L. (mastruz) obteve o maior valor de uso ( $\mathrm{VU}=1,58)$, sendo portanto, a planta com elevado potencial de uso para a comunidade. Quanto ao uso terapêutico, observou-se que um maior número de espécies foi indicado no combate à gripe, seguido por má digestão. O IR demonstrou que planta mais versátil foi Chenopodium ambrosioides $\mathrm{L}$. (mastruz), com $\mathrm{IR}=2$, referido em cinco categorias de uso e 0 $\mathrm{FCl}$ apontou que os sistemas corporais que apresentam maior importância local são: sistema circulatório $(\mathrm{FCl}=0,57)$, seguido por lesões e consequências de causas externas ( $\mathrm{FCl}=0,50)$, sistema endócrino $(\mathrm{FCl}=0,50)$ e respiratório $(\mathrm{FCl}=0,40)$. No âmbito geral, verificou-se que a diversidade de plantas medicinais conhecida e a obtenção das plantas na comunidade estudada sugerem uma correlação entre uso/conhecimento de plantas medicinais e disponibilidade das mesmas; que a flora medicinal é representada, em boa parte, por plantas exóticas cultivadas nos quintais e que a transmissão do conhecimento tradicional feito localmente e por via oral demonstra uma herança cultural na cidade.

Palavras-chave: Comunidade. Etnobotânica. Terapêuticos. APA Delta do Parnaíba.

ABSTRACT: Known medicinal plants found in the urban Cajueiro da Praia urban area, Piauí state, Northeast Brazil. The use of medicinal plants for the purposes of treating, curing and preventing diseases is considered one of the oldest forms of humanity's medical practices. This study aimed to identify the species of medicinal plants used in the Cajueiro da Praia community, Piaui state, the part of the plant used, the method of preparation, their relative importance, the use value and the consensus on the therapeutic properties of the species mentioned. 12 semi-structured interviews with indicated local experts were performed, using the "snow-ball" sampling method and the "guided tour" technique to collect the aforementioned species by a standardized method. Quantitative analyzes such as Use value, relative importance and informant consensus factor were performed alongside qualitative analyzes. 43 species belonging to 24 botanical families were cited, Lamiaceae being the most represented in number of species (eight species) followed by Euphorbiaceae, Fabaceae, Myrtaceae and Rutaceae (with three each). Chenopodium ambrosioides L. (mastruz) had the highest use value (UV $=1.58$ ), and therefore was the plant with the highest potential for use by the community. For therapeutic use, it was observed that a greater number of species have been shown to combat influenza, followed

Recebido para publicação em 28/07/2015 
by maldigestion. The RI showed that the most versatile plant was Chenopodium ambrosioides $\mathrm{L}$. (mastruz) with $\mathrm{RI}=2$, found in five usage categories and the ICF pointed out that the bodily systems that have greater local importance are: the circulatory system $(I C F=0.57)$, followed by lesions and consequences by external causes $(\mathrm{ICF}=0.50)$, the endocrine $(\mathrm{ICF}=0.50)$, and the respiratory system $(I C F=0.40)$. Within the general framework, it was found that the diversity of known medicinal plants and plants obtained from the population studied, suggested a correlation between the use/knowledge of medicinal plants and the availability there of; the medicinal flora is largely represented by exotic plants that are grown in backyards andby the transmission of traditional knowledge made locally and orally demonstrates a cultural heritage in the city.

Keywords: Urban community. Ethnobotany. Pharmacopoeia. Piauí state.

\section{INTRODUÇÃO}

Ao longo dos anos tem-se percebido que o crescimento urbano, ou a mudança de paisagem, como preferem chamar alguns autores (Buchecker et al., 2003; Mckinney, 2008), tem resultado em desenvolvimento físico e econômico. Os espaços rurais estão sendo rapidamente arquitetados para assumir a forma de ambientes urbanos.

A alteração da paisagem tem provocado mudanças também no modo de vida de diversas comunidades humanas antes relativamente isoladas, como por exemplo, os pescadores e agricultores de subsistência (Gandolfo \& Hanazaki, 2011). Sobre as implicações culturais dessa dinâmica de transformação e/ou evolução do espaço rural, Kruger \& Shannon (2000) afirmaram que a identidade cultural dessas comunidades, desenvolvida a partir da conexão cognitiva, emocional e simbólica com os recursos vegetais, não é afetada em sua intensidade, mas em seu processo.

Particularmente no caso das plantas medicinais, a influência direta da medicina ocidental moderna oportunizada pelos centros e profissionais da saúde somada à facilidade de acesso aos medicamentos alopáticos por meios das farmácias presentes nas áreas urbanas, são alguns dos fatores que afetam o conhecimento e práticas da medicina local. Apesar disso, os recursos vegetais para fins terapêuticos em áreas urbanas ainda têm sido fortemente empregados. Nestas áreas, eles são geralmente encontrados nos quintais e mercados públicos, espaços urbanos que oferecem alternativas terapêuticas mais baratas quando comparadas ao alto custo dos medicamentos alopáticos (Parente \& Rosa, 2001; Rao et al., 2004).

Estudos sobre plantas medicinais desenvolvidos em comunidades urbanas, quando comparados àqueles realizados em comunidades rurais, ainda são recentes e pontuais. Estes começaram a surgir no presente século, sendo a Argentina um dos países com um número bem representativo de estudos nesta vertente (Cuassolo et al., 2010; Hilgert et al., 2010; Pirondo et al., 2011;
Ladio et al., 2013, Rovere et al., 2013).

No Brasil, destacam-se estudos mais abrangentes em áreas urbanas os realizados por Marodin \& Baptista (2001), Fuck et al. (2005), Negrelle et al. (2007), Cruz-Silva et al. (2009), Costa \& Mayworm (2011), Gandolfo \& Hanazaki (2011), Liporacci \& Simão (2013) e Zucchi et al. (2013).

Na região Nordeste do Brasil, tais estudos ainda são escassos (Oliveira et al., 2010; Araújo et al., 2014). Recentemente, foi realizado estudo em bairro urbano da cidade de Parnaíba, norte do Piauí (Oliveira et al., 2015), no qual os autores verificaram que as plantas com potencial medicinal representam um forte recurso para a população estudada no tratamento das mais variadas enfermidades, sendo perceptível um grau bem considerável de uso.

Diante disso, e sabendo que este Estado possui comunidades urbanas localizadas dentro da Área de Proteção Ambiental (APA) Delta do Parnaíba, uma Unidade de Conservação de reconhecida importância biológica e sociocultural para o Nordeste brasileiro, este trabalho visa identificar as espécies de plantas medicinais usadas na comunidade de Cajueiro da Praia, Piauí, além de alcançar dados como a parte da planta utilizada, o modo de preparo, a sua importância relativa, o valor de uso e o consenso em relação as propriedades terapêuticas das espécies citadas.

\section{MATERIAL E MÉTODOS}

\section{Descrição da área de estudo}

Este estudo foi desenvolvido na sede do município de Cajueiro da Praia (02 $55^{\circ} 40^{\prime \prime} S$ $41^{\circ} 20^{\prime} 10^{\prime \prime}$ ), litoral norte do estado do Piauí, a 384 $\mathrm{km}$ da capital, no extremo leste da APA Delta do Parnaíba, Nordeste do Brasil.

Esta APA, que é parte de uma unidade de conservação de uso sustentável, foi criada pelo decreto federal $s / n^{\circ}$ de 28 de agosto de $1996 \mathrm{com}$ os objetivos de proteger os rios Parnaíba, Timonha e Ubatuba, melhorar a qualidade de vida das

Rev. Bras. PI. Med., Campinas, v.18, n.2, p.442-450, 2016. 
populações residentes, fomentar o turismo ecológico e a educação ambiental, além de preservar as culturas e as tradições locais.

O clima do município é tropical alternadamente úmido e seco, com temperatura média de $29^{\circ} \mathrm{C}$, possuindo formações vegetais de restinga, vegetação de dunas e caatinga arbórea e arbustiva (Aguiar, 2004).

Conforme o censo populacional realizado pelo Programa de Saúde da Família (PSF) em 2009, na sede municipal de Cajueiro da Praia residem 2.600 pessoas, das quais 1.912 tem idade a partir de 18 anos. Esta população vive, principalmente, da pesca industrial e artesanal, carcinicultura, turismo e agricultura de subsistência (BRASIL, 2002).

\section{Coleta dos dados}

Utilizando o método de amostragem não probabilístico por "bola-de-neve" foram selecionados os informantes-chave deste estudo, ou seja, aqueles que possuem maior conhecimento local sobre o uso das plantas, também chamados de especialistas locais (Albuquerque et al., 2014a). Para que os informantes citassem as plantas medicinais foi empregada a técnica da lista livre e, em seguida, foi feita uma entrevista semiestruturada (Albuquerque et al., 2014b), a fim de obter as informações específicas sobre as plantas mencionadas, tais como as indicações terapêuticas (doenças), parte da planta utilizada, estado de uso da planta, dosagem, contraindicação, formas de preparo, modos de administração, armazenamento e modos de obtenção.

Para coletar as plantas citadas nas listas livre foi feita a técnica da "turnê-guiada" (Albuquerque et al., 2014b) com os especialistas locais. O material botânico coletado foi herborizado de acordo com metodologia usual em botânica (Mori et al., 1989; Santos et al., 2014), identificado por consultas a especialistas em taxonomia botânica e por comparação com material já identificado e incorporado na coleção do Herbário "HDelta" do Campus Ministro Reis Velloso da Universidade Federal do Piauí.

A informação referente ao hábito das espécies seguiu Font-Quer (1982) e para a origem das plantas seguiu-se Lozano et al. (2014), o qual considerou as plantas originadas de fora da América do Sul como exóticas e as da América do Sul como nativas, além de consultas às obras Lorenzi \& Matos (2002, 2008) e Sousa \& Lorenzi $(2005,2012)$.

Análise dos dados

Para identificar os sistemas corporais ou categorias de doenças que gozam de maior consenso entre os informantes foi calculado o Fator de Consenso dos Informantes ( $\mathrm{FCl}$ ) proposto por Trotter \& Logan (1986). O valor máximo do $\mathrm{FCl}$ que uma categoria pode atingir é 1 , o que indica existir na comunidade um critério de seleção de plantas medicinais bem definido e/ou que as informações de uso são compartilhadas entre as pessoas. As indicações terapêuticas foram categorizadas por meio da Classificação Internacional de Doenças (CID-10) segundo a (OMS, 2003).

Para avaliar a importância do vegetal para as populações locais, ou seja, a espécie será mais importante quanto mais versátil (maior número de indicações) se apresentar, foi também calculada a Importância Relativa (IR), sendo 2 o valor máximo (Bennett \& Prance, 2000 ) que uma espécie pode obter.

Para saber qual espécie sofre maior pressão de uso foi calculado o Valor de Uso (VU), de Phillips \& Gentry (1993 a,b) e Phillips et al. (1994), modificada por Rossato et al. (1999).

\section{RESULTADOS E DISCUSSÃO}

Foram citadas 43 espécies de plantas para uso medicinal, as quais estão distribuídas em 36 gêneros e 24 famílias (Tabela 1), sendo Lamiaceae a de maior representatividade, com oito espécies. Este último dado também foi encontrado em outros estudos (Christo et al., 2006; Teixeira \& Melo, 2006, Costa \& Mayworm, 2011; Alves \& Povh, 2013), o que pode ser explicado por sua distribuição cosmopolita e grande importância terapêutica de suas espécies (Di Stasi et al., 2002; Pinto et al., 2006; Alves \& Povh, 2013). De fato, Judd et al. (2009) traz muitas espécies desta família como sendo ricas em óleos essenciais, atribuindo-as propriedades aromáticas e medicinais.

Das espécies utilizadas como fitoterápicas na comunidade, 32 são exóticas e cultivadas nos quintais enquanto 11 são nativas. Este predomínio de plantas exóticas talvez se deva ao hábito de se cultivar, para uso próprio, as plantas em seus quintais para fins alimentícios ou medicinais, fato frequente nas populações. Aguiar \& Barros (2012) relataram que os quintais são locais de teste e seleção, além de acúmulo de conhecimento em relação ao uso de plantas. Na comunidade estudada, a troca de mudas de plantas entre vizinhos e amigos é a forma mais frequente de propagação das plantas medicinais, pois espécies nativas não encontradas nos quintais de um entrevistado são encontradas em quintais de outros, o que pode ser explicado o fato de existir um maior número de espécies exóticas em detrimento das nativas.

As plantas medicinais citadas possuem, em sua maioria, hábito do tipo herbáceo (15 spp.), seguido pelos hábitos arbóreo (16 spp.), arbustivo (7 spp.), subarbustivo (4 spp.) e trepador (1 spp.). Segundo Neto et al. (2014), plantas herbáceas são

Rev. Bras. PI. Med., Campinas, v.18, n.2, p.442-450, 2016. 
TABELA 1. Lista das plantas medicinais citadas por especialistas locais da zona urbana de Cajueiro da Praia, Piauí, Nordeste do Brasil. $\mathrm{S}$ = status: $\mathrm{n}$ = nativa, $\mathrm{e}=$ exótica; $\mathrm{VU}=$ Valor de Uso; IR=importância relativa; $\mathrm{NC}=$ Número de coleta do primeiro autor.

\begin{tabular}{|c|c|c|c|c|c|c|c|}
\hline FAMILIA/ESPÉCIE & Nome Popular & Hábito & $\mathrm{S}$ & Indicação Terapêutica & VU & IR & NC \\
\hline \multicolumn{8}{|l|}{ ACANTHACEAE } \\
\hline 1. Justicia pectoralis Jacq. & Trevo & Erva & $E$ & $\begin{array}{c}\text { Dor de barriga/cólica } \\
\text { menstrual/ má digestão }\end{array}$ & 0,25 & 0,77 & 02 \\
\hline \multicolumn{8}{|l|}{ AMARANTHACEAE } \\
\hline 2. Alternanthera dentata (Moench) & penicilina & erva & $\mathrm{n}$ & Inflamação em geral & 0,08 & 0,32 & 48 \\
\hline \multicolumn{8}{|l|}{ Stuchlik ex R.E.Fr. } \\
\hline 3. Beta vulgaris L. & beterraba & erva & e & Anemia & 0,16 & 0,32 & 51 \\
\hline \multicolumn{8}{|l|}{ ANACARDIACEAE } \\
\hline 4. Anacardium occidentale L. & Caju & árvore & $\mathrm{n}$ & Ferimento & 0,08 & 0,32 & 31 \\
\hline 5. Mangifera indica L. & mangueira & árvore & $\mathrm{e}$ & Coriza & 0,08 & 0,32 & 28 \\
\hline \multicolumn{8}{|l|}{ ANNONACEAE } \\
\hline 6. Annona muricata L. & Graviola & árvore & $\mathrm{n}$ & Diabete & 0,08 & 0,32 & 40 \\
\hline 7. Annoa squamosa L. & Ata & árvore & $\mathrm{e}$ & Má digestão & 0,08 & 0,32 & 30 \\
\hline \multicolumn{8}{|l|}{ APOCYNACEAE } \\
\hline 8. Catharanthus roseus (L.) G. Don & boa noite & árvore & $\mathrm{e}$ & Dor no corpo & 0,08 & 0,32 & 01 \\
\hline \multicolumn{8}{|l|}{ CAPPARACEAE } \\
\hline 9. Crataeva tapia L. & trapiá & árvore & $\mathrm{n}$ & Hemorróida & 0,08 & 0,32 & 06 \\
\hline \multicolumn{8}{|l|}{ CARICACEAE } \\
\hline 10. Carica papaya L. & mamão & árvore & $\mathrm{n}$ & Hérnia & 0,08 & 0,32 & 39 \\
\hline \multicolumn{8}{|l|}{ CHENOPODIACEAE } \\
\hline 11. Chenopodium ambrosioides L. & mastruz & subarbusto & e & $\begin{array}{c}\text { Ferimento/pancada/gripe/ } \\
\text { pneumonia/ enfraquecimento/ } \\
\text { sangramento/gastrite/ } \\
\text { inflamação em geral/ }\end{array}$ & 1,58 & 2,00 & $11,22,36$ \\
\hline \multicolumn{8}{|l|}{ CRASSULACEAE } \\
\hline 12. Bryophyllum pinnatum (Lam.) Oken & corama & erva & e & $\begin{array}{c}\text { Gastrite/inflamação/ } \\
\text { sangramento/gripe/tosse }\end{array}$ & 0,41 & 1,22 & 41 \\
\hline \multicolumn{8}{|l|}{ CUCURBITACEAE } \\
\hline 13. Momordica charantia L. & $\begin{array}{l}\text { melão-são- } \\
\text { caetano }\end{array}$ & trepadeira & e & $\begin{array}{l}\text { Infecção na pele e pano } \\
\text { branco }\end{array}$ & 0,08 & 0,32 & 50 \\
\hline \multicolumn{8}{|l|}{ EUPHORBIACEAE } \\
\hline 14. Croton sonderianus Müll. Arg. & marmeleiro & árvore & $\mathrm{n}$ & Má digestão/inflamação & 0,33 & 0,65 & 17 \\
\hline 15. Phyllanthus niruri L. & quebra-pedra & erva & $n$ & $\begin{array}{c}\text { Dor nos rins/figado/diabete/ } \\
\text { uretra }\end{array}$ & 0,25 & 0,97 & 16,38 \\
\hline \multicolumn{7}{|l|}{ FABACEAE (Subfamília } & 58 \\
\hline \multicolumn{8}{|l|}{ Caesalpinioideae) } \\
\hline 17. Bauhinia unguiculata Baker & pata-de-vaca & arbusto & $\mathrm{n}$ & Diabete & 0,08 & 0,32 & 55 \\
\hline 18. Caesalpinia gardneriana Benth. & catingueira & arbusto & $\mathrm{n}$ & Gripe/tosse & 0,25 & 0,45 & 08 \\
\hline 19. Tamarindus indica L. & tamarindo & árvore & e & Hemorróida & 0,08 & 0,32 & 45 \\
\hline \multicolumn{8}{|l|}{ IRIDACEAE } \\
\hline \multicolumn{8}{|l|}{ LAMIACEAE } \\
\hline 21. Mentha arvensis L. & hortelã-vick & erva & e & Gripe/asma & 0,25 & 0,45 & 03 \\
\hline 22. Plectranthus amboinicus (Lour.) & malva & erva & e & Gripe/tosse/febre/inflamação & 1,08 & 0,90 & 12,34 \\
\hline Spreng. & & & & em geral & & & \\
\hline 23. Plectranthus barbatus Andrews & boldo-grande & erva & e & Má digestão/ressaca & 0,25 & 0,65 & 15 \\
\hline 24. Plectranthus neochilus Schtr. & Boldo-pequeno & erva & $\mathrm{e}$ & $\begin{array}{l}\text { Má digestão/fígado/prisão de } \\
\text { ventre/dor de cabeça }\end{array}$ & 0,33 & 0,90 & $13,23,46$ \\
\hline 25. Mentha $x$ villosa Huds. & hortelã & erva & e & $\begin{array}{c}\text { Dor de barriga/má digestão/ } \\
\text { gripe/tosse }\end{array}$ & 0,75 & 0,90 & 32 \\
\hline 26. Mentha arvensis L. & Vick & erva & e & Febre/gripe/tosse & 0,25 & 0,77 & 33 \\
\hline
\end{tabular}

Rev. Bras. PI. Med., Campinas, v.18, n.2, p.442-450, 2016. 
TABELA 1. Lista das plantas medicinais citadas por especialistas locais da zona urbana de Cajueiro da Praia, Piauí, Nordeste do Brasil. $\mathrm{S}$ = status: $\mathrm{n}=$ nativa, e = exótica; $\mathrm{VU}=$ Valor de Uso; IR=importância relativa; $\mathrm{NC}=$ Número de coleta do primeiro autor.

\begin{tabular}{|c|c|c|c|c|c|c|c|}
\hline \multirow[b]{2}{*}{ 27. Ocimum basilicum L. } & \multirow[b]{2}{*}{ manjericão } & \multirow{3}{*}{$\begin{array}{c}\text { erva } \\
\text { subarbusto }\end{array}$} & \multirow[b]{2}{*}{ e } & \multirow[b]{2}{*}{ Gripe } & \multirow[b]{2}{*}{0,08} & \multicolumn{2}{|c|}{ 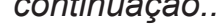 } \\
\hline & & & & & & 0,32 & 35 \\
\hline 28. Hyptis atrorubens Poit. & erva-cidreira & & e & $\begin{array}{c}\text { Pressão/estresse/tosse/dor } \\
\text { de cabeça/azia }\end{array}$ & 0,66 & 1,62 & 37 \\
\hline \multicolumn{8}{|l|}{ LAURACEAE } \\
\hline $\begin{array}{l}\text { 29. Persea americana Mill. } \\
\text { LILIACEAE }\end{array}$ & abacate & árvore & e & Dor nos rins & 0,08 & 0,32 & 47 \\
\hline 30. Aloe sp. & $\begin{array}{l}\text { babosa- } \\
\text { pequena }\end{array}$ & erva & e & $\begin{array}{l}\text { Próstata/gastrite/câncer/ } \\
\text { inflamação em geral }\end{array}$ & 0,33 & 1,30 & 18,25 \\
\hline $\begin{array}{l}\text { 31. Aloe vera (L.) Burm. f. } \\
\text { MALPIGHIACEAE }\end{array}$ & \multicolumn{6}{|c|}{ MALPIGHIACEAE } & 44 \\
\hline \multicolumn{7}{|l|}{ MALVACEAE } & 26 \\
\hline \multicolumn{8}{|l|}{ MUSACEAE } \\
\hline $\begin{array}{l}\text { 34. Musa paradisiaca L. } \\
\text { MYRTACEAE }\end{array}$ & bananeira & arbusto & e & Diarréia & 0,08 & 0,32 & 43 \\
\hline 35. Eucalyptus globulus Labill. & eucalipto & árvore & e & Febre/coriza/asma/diarréia & 0,58 & 1,10 & 7 \\
\hline 36. Psidium guajava L. & goiaba & árvore & $\mathrm{n}$ & Diarréia & 0,25 & 0,32 & $5,29,53$ \\
\hline $\begin{array}{l}\text { 37. Syzygium cumini (L.) Skeels } \\
\text { POACEAE }\end{array}$ & azeitona-roxa & árvore & e & Gastrite & 0,08 & 0,32 & 57 \\
\hline 38. Cymbopogon citratus Stapf. & capim-limão & árvore & e & $\begin{array}{c}\text { Estresse/gripe/pressão /má } \\
\text { digestão/pneumonia }\end{array}$ & 0,75 & 1,42 & 9,24 \\
\hline \multicolumn{8}{|l|}{ PUNICACEAE } \\
\hline \multicolumn{8}{|l|}{ RUTACEAE } \\
\hline 40. Critus aurantium L. & laranjeira & árvore & e & $\begin{array}{c}\text { Coração/má digestão/ } \\
\text { estresse }\end{array}$ & 0,25 & 0,97 & 42 \\
\hline 41. Critus limon L. Burm. f. & limoeiro & árvore & e & Gripe & 0,16 & 0,32 & 20 \\
\hline 42. Ruta graveolens L. & arruda & subarbusto & e & $\begin{array}{c}\text { Dor de barriga/má digestão/ } \\
\text { gastrite }\end{array}$ & 0,25 & 0,57 & 56 \\
\hline \multicolumn{8}{|l|}{ SOLANACEAE } \\
\hline 43. Lycopersicon esculentum Mill. & tomate & subarbusto & $\mathrm{E}$ & Febre & 0,08 & 0,32 & 10,52 \\
\hline
\end{tabular}

fáceis de serem cultivadas em quintais e hortas. Embora todas as partes das plantas tenham sido mencionadas, as partes mais citadas para usos medicinais foram as folhas, seguida das cascas e frutos.

As folhas são utilizadas principalmente para a preparação de chás, por decocção ou infusão, dependendo da parte da planta que é utilizada. Esta significativa maioria do uso de folhas deve-se, provavelmente, à frequente utilização de plantas medicinais herbáceas pelas pessoas da comunidade. Aguiar \& Barros (2012) mencionam que as plantas herbáceas são localizadas em locais restritos, como os quintais, assim, percebe-se existir uma relação entre os fatos de as plantas exóticas serem as mais citadas e o hábito herbáceo ser o mais encontrado.

Resultado análogo para a utilização de folhas foi observado nos trabalhos de Chaves (2005) em Cocal (PI), Fuck et al. (2005) em Bandeirantes
(PR) e Franco \& Barros (2006) no Quilombo Olho D'água dos Pires, Esperantina (PI). Entretanto, Almeida (2004), estudando o uso de plantas medicinais em áreas de caatinga, observou que as partes mais citadas foram as flores, seguida pelas folhas e entrecascas do caule. Conforme informações dos entrevistados na área estudada, a maioria das partes das plantas utilizadas como remédios podem ser obtidas em qualquer época do ano, principalmente devido ao fato de as partes mais usadas serem as folhas, que estão presentes na maioria das plantas durante $o$ ano todo.

Foram citadas três maneiras diferentes de preparo de chás, estas já classificadas segundo Simões (1998) em: infusão, decocção e maceração. Segundo Kffuri (2008), a forma de preparo é importante na conservação adequada dos princípios ativos das plantas medicinais e consequentemente a sua eficácia terapêutica.

Ainfusão foi a forma de preparo mais utilizada

Rev. Bras. PI. Med., Campinas, v.18, n.2, p.442-450, 2016. 
TABELA 2. Fator de Consenso dos Informantes (FCl) quanto aos sistemas corporais ou categorias de doenças tratadas por plantas medicinais na comunidade de Cajueiro da Praia, Piauí, Nordeste do Brasil. CID: Classificação Internacional de Doenças, segundo a Organização Mundial de Saúde-OMS (2003).

\begin{tabular}{|c|c|c|}
\hline INDICAÇÕES TERAPÊUTICAS & CATEGORIAS DE DOENÇAS CONFORME CID-10 & $\mathrm{FCl}$ \\
\hline $\begin{array}{l}\text { Pressão } \\
\text { Coração }\end{array}$ & Doenças do aparelho circulatório & 0,57 \\
\hline Pancada & $\begin{array}{l}\text { Lesões, envenenamento e algumas outras consequências de } \\
\text { causas externas }\end{array}$ & 0,50 \\
\hline Diabete & Doenças endócrinas, nutricionais e metabólicas & 0,50 \\
\hline $\begin{array}{l}\text { Asma } \\
\text { Gripe } \\
\text { Tosse } \\
\text { Garganta } \\
\text { Pneumonia } \\
\text { Coriza }\end{array}$ & Doenças do aparelho respiratório & 0,40 \\
\hline $\begin{array}{l}\text { Fígado } \\
\text { Gastrite } \\
\text { Dor no estômago } \\
\text { Má digestão } \\
\text { Diarréia } \\
\text { Azia } \\
\text { Dor de barriga } \\
\text { Hérnia } \\
\text { Hemorróida } \\
\text { Prisão de ventre }\end{array}$ & Doenças, sintomas e sinais relativos ao aparelho digestivo e abdome & 0,33 \\
\hline $\begin{array}{l}\text { Febre } \\
\text { Inflamação em geral, } \\
\text { Dor de cabeça } \\
\text { Enfraquecimento } \\
\text { Dor no corpo } \\
\text { Ressaca } \\
\text { Sangramento }\end{array}$ & Sintomas e sinais gerais & 0,33 \\
\hline $\begin{array}{l}\text { Cólica menstrual } \\
\text { Problemas nos rins e uretra } \\
\text { Próstata }\end{array}$ & Doenças do aparelho geniturinário & 0,33 \\
\hline Estresse & Transtornos mentais e comportamentais & 0,33 \\
\hline $\begin{array}{l}\text { Pano branco } \\
\text { Infecção na pele } \\
\text { Ferimento } \\
\text { Queda de cabelo }\end{array}$ & Doenças da pele e do tecido subcutâneo & 0,16 \\
\hline Câncer & Neoplasias & 0,00 \\
\hline Anemia & Doenças do sangue e dos órgãos hematopoéticos & 0,00 \\
\hline
\end{tabular}

na comunidade. Entretanto, em outras regiões do país, Medeiros et al. (2004) em Mangaratiba (RJ); Pinto et al. (2006) em comunidades rurais de Itacaré (BA); Oliveira (2008) no semiárido piauiense e Giraldi (2009) em Florianópolis (SC) verificaram a decocção como a forma de preparo mais frequente, seguida da infusão. Além dos chás, as preparações medicinais são feitas também na forma de lambedor, banho, garrafada, sucos e outros. Também verificou-se na área de estudo que é uma prática bastante comum o uso em associações de várias espécies na produção de lambedores e garrafadas.

Segundo os entrevistados, o modo de administração das plantas medicinais se dá em grande parte de forma interna (via oral). Amorozo (2002), Franco \& Barros (2006), Pinto et al. (2006), Maioli-Azevedo \& Fonseca-Kruel (2007), Oliveira (2008) e Giraldi (2009), também constataram que este é o modo de administração mais frequente.

As doenças foram categorizadas de acordo com o CID-10-Classificação estatística internacional de doenças e problemas relacionados à saúde (OMS, 2003) (Tabela 2).

As categorias de doença ou indicações terapêuticas que atingiram o maior valor no $\mathrm{FCl}$ foram aquelas relacionadas a doenças do aparelho circulatório (pressão arterial), doenças endócrinas (diabete), lesões (pancadas) e doenças do aparelho 
respiratório (gripe) (Tabela 2).

As espécies que apresentaram maior versatilidade de usos, verificada através do cálculo de Importância Relativa (IR), foram o mastruz (Chenopodium ambrosioides, IR=2), ervacidreira (Hyptis atrorubens, IR=1,62), capim-limão (Cymbopogon citratus, IR=1,42), babosa-pequena (Aloe sp., IR=1,30) e eucalipto (Eucalyptus globulus, $\mathrm{IR}=1,10)$. Chenopodium ambrosioides $\mathrm{L}$. é uma planta herbácea com ampla distribuição no território brasileiro, conhecida popularmente como mastruço, mastruz, erva-de-santa-maria, chá-do-méxico, erva-formigueira e quenopódio (Lorenzi \& Matos, 2008) empregada no tratamento de lesões cutâneas causadas por Leishmania (Viannia) braziliensis (França et al., 1996) e alívio da dor de barriga e da gripe (Moreira et al., 2002). Pelos entrevistados, Chenopodium ambrosioides $\mathrm{L}$. serve para ferimento, pancada, gripe, pneumonia, enfraquecimento, sangramento, gastrite e inflamação em geral, o que coincide com o estudo de Viegas et al. (2014), no qual os entrevistados a usavam para o tratamento de gastrite e o de Baptistel et al. (2014) onde a mesma foi citada para luxações, machucados em geral, puerpério, vermes, ferimentos inflamados, gastrite, fraturas e hematomas (pancadas).

As espécies que obtiveram os maiores VU foram matruz (Chenopodium ambrosioides, $\mathrm{VU}=$ 1,58 ) e malva (Plectranthus amboinicus, $\mathrm{VU}=1,08$ ), já que são usadas para diferentes enfermidades. Esta última foi indicada pela comunidade para gripe, tosse, febre e inflamação em geral. Estas duas espécies também foram registradas em outros trabalhos (Feijó et al., 2013; Liporacci \& Simão, 2013; Zucchi et al., 2013)).

\section{CONSIDERAÇÕES FINAIS}

Esta pesquisa permitiu identificar alguns aspectos relevantes sobre o uso e o conhecimento de plantas medicinais na cidade de Cajueiro da Praia, Piauí: a diversidade de plantas medicinais conhecida e a obtenção das plantas na própria comunidade sugerem uma correlação entre uso/conhecimento de plantas medicinais e disponibilidade das mesmas; a flora medicinal é representada, em boa parte, por plantas exóticas cultivadas nos quintais; as nativas foram menos representativas.

Este levantamento das espécies vegetais utilizadas para fins medicinais no município de Cajueiro da Praia contribuiu com a expansão de informações e/ou complementação no estado do Piauí, pois permitiu elaborar um check-list das espécies vegetais usadas no município estudado, permitiu também identificar as enfermidades e os sistemas corporais tratados, que serão citados e/ou usados pelos informantes, alcançando, em última instância, dados que irão proporcionar o incentivo à aplicabilidade dos recursos naturais vegetais na região, focalizando o desenvolvimento sustentável com consequente melhoria na qualidade de vida da população.

Ainda, no âmbito geral, este estudo permitiu verificar que o uso de plantas medicinais assume grande valor na vida destas pessoas, pois, em virtude deste e de outros fatores, muitas famílias estão cada vez mais estimuladas a utilizá-las.

\section{REFERÊNCIAS}

AGUIAR, L.C.G.G.; BARROS, R.F.M. Plantas medicinais cultivadas em quintais de comunidades rurais no domínio do cerrado piauiense (Município de Demerval Lobão, Piauí, Brasil). Revista Brasileira de Plantas Medicinais, v.14, n.3, p.419-434, 2012.

AGUIAR, R.B.A. Projeto cadastro de fontes de abastecimento por água subterrânea, estado do Piauí: diagnóstico do município de Cajueiro da Praia. Fortaleza: CPRM - Serviço Geológico do Brasil, 2004. 9p. (Relatório Técnico).

ALBUQUERQUE, U.P., LUCENA, R.F.P., LINS NETO, E.M.F. Selection of Research Participants. In: ALBUQUERQUE, U.P., CUNHA, L.V.F.C., LUCENA, R.F.P., ALVES, R.R.N. (Eds.). 1.ed. Methods and techniques in ethnobiology and ethnoecology. New York, USA: Springer, 2014a, pp. 1-13.

ALBUQUERQUE, U.P., RAMOS, M.A., LUCENA, R.F.P., ALENCAR, N.L. Methods and techniques used to collect ethnobiological data. In: ALBUQUERQUE, U.P., CUNHA, L.V.F.C., LUCENA, R.F.P., ALVES, R.R.N. (eds.). Methods and techniques in ethnobiology and ethnoecology. 1.ed. New York, USA: Springer, 2014b, pp. 15-37.

ALMEIDA, C.F.C.B.R. Etnobotânica nordestina: estratégia de vida e composição química como preditores do uso de plantas medicinais por comunidades locais na caatinga. 2004. $66 \mathrm{f}$. Dissertação (Mestrado em Biologia Vegetal) Universidade Federal de Pernambuco, Recife.

ALVES, G. S. P; POVH, J. P.; Estudo etnobotânico de plantas medicinais na comunidade de Santa Rita, Ituiutaba-MG. Biotemas, v. 26, n. 3, p. 231-242, 2013.

AMOROZO, M.C.M. Uso e diversidade de plantas medicinais em Santo Antonio do Leverger, MT, Brasil. Acta Botanica Brasilica, v.16, n.2, p.189-203, 2002.

ARAÚJO, C.R.F. et al. Perfil e prevalência de uso de plantas medicinais em uma unidade básica de saúde da família em Campina Grande, Paraíba, Brasil. Revista de Ciências Farmacêuticas Básica e Aplicada, v.35, n.2, p.233-238, 2014.

BAPTISTEL, A.C. et al. Plantas medicinais utilizadas na Comunidade Santo Antônio, Currais, Sul do Piauí: um enfoque etnobotânico. Revista Brasileira de Plantas Medicinais, v.16, n.2, supl. I, p.406-425, 2014.

BENNETT, B.C. ; PRANCE, G.T. Introduced plants in the indigenous pharmacopoeia of Northern South America. Economic Botany, v.54, n.1 p.90-102, 2000. 
BRASIL. Zoneamento Ecológico-Econômico do Baixo Parnaíba. Subsídios técnicos, Relatório Final. BrasíliaDF: MMA/SDS. 2002, 92p.

BUCHECKER, M. et al. Participatory landscape development: overcoming social barriers to public involvement. Landscape Urban Plants, v. 64 , p. 2946, 2003.

CHAVES, E.M.F. Florística e potencialidades econômicas da vegetação de carrasco no município de Cocal, Piauí, Brasil. Teresina: UFPI, 2005. 112f. Dissertação (Mestrado em Desenvolvimento e Meio Ambiente) - Programa de Pós-Graduação em Desenvolvimento e Meio Ambiente. Universidade Federal do Piauí, Teresina.

CHRISTO, A.G.; et al., Uso de recursos vegetais em comunidades rurais limítrofes à Reserva Biológica de Poço das Antas, Silva Jardim, Rio de Janeiro: Estudo de caso na Gleba Aldeia Velha. Rodriguésia, v.57, n.3, p.195-206, 2006.

COSTA, V. P.; MAYWORM, M. A. S. Plantas medicinais utilizadas pela comunidade do bairro dos Tenentes município de Extrema, MG, Brasil. Revista Brasileira de Plantas Medicinais,n. 3, v. 13, p. 282-292, 2011.

CRUZ-SILVA, C. T. A. et al. Abordagem Etnobotânica acerca do uso de plantas medicinais na região urbana no município de Quedas do Iguaçu, Paraná. Cultivando - Saber, v. 2, n. 1, p. 14-25, 2009.

CUASSOLO, F. et al. Aspectos de la comercialización y control de calidad de las plantas medicinales más vendidas en una comunidad urbana del NO de la Patagonia Argentina. Boletin Latinoamericano y del Caribe de Plantas Medicinales y Aromaticas, v.9, n.3, p.166-176, 2010.

DI STASI, L.C. et al. Medicinal plants popularly used in the Brazilian Tropical Atlantic Forest. Fitoterapia, n.73, p.69-91, 2002.

FEIJÓ, E.V.R.S. et al. Levantamento preliminar sobre plantas medicinais utilizadas no bairro salobrinho no município de Ilhéus, Bahia. Revista Brasileira de Plantas Medicinais, v.15, n.4, p.595-604, 2013.

FONT-QUER, M.P. Diccionario de botanica. 8. reimp. Barcelona: Labor, 1982. 1244p.

FRANCO, E.A.P.A.; BARROS, R.F.M. Uso e diversidade de plantas medicinais no Quilombo Olho D'água dos Pires, Esperantina, Piauí, Brasil. Revista Brasileira de Plantas Medicinais, v.8, n.3, p.78-88, 2006.

FRANÇA, F. et al. Plants used in the treatment of leishmanial ulcers due to Leishmania (Viannia) braziliensis in an endemic area of Bahia, Brazil. Revista da Sociedade Brasileira de Medicina Tropical, v.29, p.229-232, 1996.

FUCK, S.B. et al. Plantas medicinais utilizadas na medicina popular por moradores da zona urbana de Bandeirantes, PR, Brasil. Semina, v.26, n.3, p.291-296, 2005.

GIRALDI, M. Uso e conhecimento tradicional de plantas medicinais no Sertão do Ribeirão, Florianópolis, SC, Brasil. 2009. 60p. Trabalho de Conclusão de Curso (Monografia de Graduação) - Universidade Federal de Santa Catarina, Florianópolis.

GANDOLFO, E.S.; HANAZAKI, N. Etnobotânica e urbanização: conhecimento e utilização de plantas de restinga pela comunidade nativa do distrito do Campeche (Florianópolis, SC). Acta Botanica
Brasilica, v. 25, n.1, p.168-177, 2011.

HILGERT, N.I. et al. La medicina herbolaria en el contexto urbano. Estudio de caso en un barrio de la ciudad de Tandil, Argentina. Boletin Latinoamericano y del Caribe de Plantas Medicinales y Aromaticas, v.9, n.3, p.177-190, 2010.

JUDD, W.S.; CAMPBELL, C.S.; KELLOG, E.A.; STEVENS, P.F.; DONOGHUE, M.J. Sistemática vegetal: um enfoque filogenético. 3. ed. Porto Alegre: Artmed, Porto Alegre, 2009. 632p.

KRUGER, L.E.; SHANNON, M.A. Getting to know ourselves and our places through participation in civic social assessment. Society and Natural Resources, v.13, n.5, p. 461-478, 2000.

KFFURI, C.W. Etnobotânica de plantas medicinais no município de Senador Firmino (Minas Gerais). 2008. 88p. Dissertação (Mestrado em Fitotecnia) Universidade Federal de Viçosa, Viçosa.

LADIO, A.H. et al. Etnobotánica aplicada en Patagonia: la comercialización de malezas de uso comestible y medicinal en una feria urbana de San Carlos de Bariloche (Río Negro, Argentina). Boletin Latinoamericano y del Caribe de Plantas Medicinales y Aromaticas, v.12, n.1, p.24-37, 2013.

LIPORACCI, H. S. N.; SIMÃO, D. G. Levantamento etnobotânico de plantas medicinais nos quintais do Bairro Novo Horizonte, Ituiutaba, Revista Brasileira de Plantas Medicinais, v. 15, n. 4, p. 529-540, 2013.

LORENZI, H.; MATOS, F. J. A. Plantas medicinais no Brasil: nativas e exóticas. 1.ed. Nova Odessa: Instituto Plantarum, 2002. $512 \mathrm{p}$.

LORENZI, H.; MATOS, F. J. A. Plantas medicinais no Brasil: nativas e exóticas. 2.ed. Nova Odessa: Instituto Plantarum, 2008. $544 \mathrm{p}$

LOZANO, A. et al. The apparency hypothesis applied to a local pharmacopoeia in the Brazilian northeast. Journal of Ethnobiology and Ethnomedicine, v.10, n.2, p. 1-17, 2014.

MAIOLI-AZEVEDO, V.; FONSECA-KRUEL, V.S.da. Plantas medicinais e ritualísticas vendidas em feiras livres no Município do Rio de Janeiro, RJ, Brasil: estudo de caso nas zonas Norte e Sul. Acta Botanica Brasilica, v.21, n.2, p.263-275, , 2007.

MARODIN, S.M.; BAPTISTA, L.R.M. O uso de plantas com fins medicinais no município de Dom Pedro de Alcântara, Rio Grande do Sul, Brasil. Revista Brasileira de Plantas Medicinais, v.4, n.1, p.57-68, 2001.

MCKINNEY, M. L. Effects of urbanization on species richness: A review of plants and animals. Urban Ecosystem, v.11, p.161-176, 2008.

MEDEIROS, M.F.T. et al. Plantas medicinais e seus usos pelos sitiantes da Reserva Rio das Pedras, Mangaratiba, RJ, Brasil. Acta Botanica Brasilica, v.18, n.2, p.391399, 2004.

MOREIRA, R. de C.T. et al. Abordagem etnobotânica acerca do uso de plantas medicinais na Vila Cachoeira, Ilhéus, Bahia, Brasil. Acta Farmacéutica Bonaerense, v.21, p.205-211, 2002.

MORI, S.A., SILVA, L.A.M., CORADIM, L. Manual de manejo do herbário fanerogâmico. 2.ed. Bahia: Centro de Pesquisa do Cacau. 1989. 103p.

NEGRELLE, R.R.B. et al. Estudo etnobotânico junto à Unidade Saúde da Família Nossa Senhora dos

Rev. Bras. PI. Med., Campinas, v.18, n.2, p.442-450, 2016. 
Navegantes: subsídios para o estabelecimento de programa de fitoterápicos na Rede Básica de Saúde do município de Cascavel (Paraná). Revista Brasileira de Plantas Medicinais, v.9, n.3, p.6-22, 2007.

NETO, F.R.G. et al. Estudo etnobotânico de plantas medicinais utilizadas pela comunidade do Sisal no município de Catu, Bahia, Brasil. Revista Brasileira de Plantas Medicinais, v.16, n.4, p. 856-865, 2014.

OLIVEIRA, F.C.S.de. Conhecimento botânico tradicional em comunidades rurais do semi-árido piauiense. 2008. 134f. Dissertação (Mestrado em Desenvolvimento e Meio Ambiente) - Programa de Pós-Graduação em Desenvolvimento e Meio Ambiente, Universidade Federal do Piauí, Teresina.

OMS - ORGANIZAÇÃO MUNDIAL DE SAÚDE. CID 10, tradução do Centro Colaborador da OMS para a Classificação de Doenças em Português. 9. ed. Revisada. São Paulo: EDUSP, 2003.

PARENTE, C.E.T.; ROSA, M.M.T.da. Plantas comercializadas como medicinais no município de Barra do Piraí, RJ. Rodriguésia, v.52, p.47-59, 2001.

PINTO, E.P.P. et al. Conhecimento popular sobre plantas medicinais em comunidades rurais de Mata Atlântica Itacaré, BA, Brasil. Acta Botanica Brasilica, v.20, n.4, p.751-762, 2006.

PIRONDO, A. et al. Influencia de factores externos sobre la comercialización de plantas medicinales en un medio urbano: el caso de vendedores criollos e indígenas en Corrientes, Argentina. Boletin Latinoamericano y del Caribe de Plantas Medicinales y Aromaticas, v.10, n.6, p.553-569, 2011.

PHILLIPS, O.; GENTRY, A.H. The useful Plants of Tamboapata, Peru: I Statistical hypothesis testing with a new quantitative technique. Economic Botany. v. 47. n.1, p. 15-32, 1993 a.

PHILLIPS, O.; GENTRY, A. H .The useful Plants of Tamboapata, Peru: II Additional hypothesis testing in quantitative ethnobotany. Economic Botany . v. 47. n. 1, p. 33-43, 1993 b.

PHILLIPS, O. et al. Quantitative ethnobotany and amazonian conservation. Conservation Biology . v.8, p. 15-32, 1994.

RAO, M.R. et al. Medicinal and aromatic plants in agroflorestry systems. Agroflorestry Systems, n.61, p.107-122, 2004.

ROSSATO, S.C. et al. Ethnobotany of Caiçaras of the Atlantic Forest Coast (Brazil). Economic Botany, n.53, p.387-395, 1999.

ROVERE, A.E. et al. Plantas utilizadas en cercos vivos de ciudades patagónicas: aportes de la etnobotánica para la conservación. Ecología Austral, v.23, p.165173, 2013.

SANTOS, L.L.; VIEIRA, F.J.; NASCIMENTO, L.G.S.; SILVA, A.C.O.; SOUZA, G.M. Techniques for collecting and processing plant material and their application in ethnobotany research. In: ALBUQUERQUE, U.P., CUNHA, L.V.F.C., LUCENA, R.F.P., AND ALVES, R.R.N. Methods and techniques in ethnobiology and ethnoecology. 1.ed. New York: Springer, 2014, 161-174.

SIMÕES, C.M.O. Plantas da Medicina Popular no Rio Grande do Sul. 5.ed. Porto Alegre: Ed. da UFRGS, 1998, 173p.

SOUSA, V.C; LORENZI, H. Botânica sistemática: guia ilustrado para identificação das famílias de Angiospermas da flora brasileira, baseado na APG II. 1.ed. Nova Odessa: Instituto Plantarum, 2005. 640 p.

SOUSA, V.C; LORENZI, H. Botânica sistemática: guia ilustrado para identificação das famílias de Fanerógamas nativas e exóticas no Brasil, baseado na APG III. 3.ed. Nova Odessa: Instituto Plantarum, 2012, 768 p.

TEIXEIRA, S.A.; MELO, J.I.M. Plantas medicinais utilizadas no município de Jupi, Pernambuco, Brasil. Iheringia, Série Botânica, v.61, n.1-2, p.5-11, 2006.

TROTTER, R.; LOGAN, M. Informant consensus: a new approach for identifying potentialli effective medicinal plants. In: ETKIN, N.L. (Ed.). Indigenous medicine and diet: behavioural approaches. 1.ed. New York: Redgrave Bedford Hills, 1986, p. 91-112.

VIEGAS, C.F. et al. Abordagem Etnobotânica em uma empresa do Polo Industrial de Manaus. Scientia Amazonia, v. 3, n.3, p.51-58, 2014.

ZUCCHI, M.R. et al. Levantamento de plantas medicinais na cidade de Ipameri-GO. Revista Brasileira de Plantas Medicinais, v.15, n.2, p.273-279, 2013. 